\title{
Automated Environment Generation for Software Model Checking
}

\author{
Oksana Tkachuk, Matthew B. Dwyer \\ Department of CIS \\ Kansas State University, USA \\ oksana@cis.ksu.edu
}

\author{
Corina S. Păsăreanu \\ Kestrel Technologies \\ Moffett Field, USA \\ pcorina@email.nasa.arc.gov
}

\begin{abstract}
A key problem in model checking open systems is environment modeling (i.e., representing the behavior of the execution context of the system under analysis). Software systems are fundamentally open since their behavior is dependent on patterns of invocation of system components and values defined outside the system but referenced within the system. Whether reasoning about the behavior of whole programs or about program components, an abstract model of the environment can be essential in enabling sufficiently precise yet tractable verification.

In this paper, we describe an approach to generating environments of Java program fragments. This approach in tegrates formally specified assumptions about environment behavior with sound abstractions of environment implementations to form a model of the environment. The approach is implemented in the Bandera Environment Generator (BEG) which we describe along with our experience using $B E G$ to reason about properties of several non-trivial concurrent Java programs.
\end{abstract}

\section{Introduction}

Model checking the source code of realistic software systems is a challenge and is currently the topic of a large number of research efforts (e.g., [7, 16, 30]). The primary challenge lies in overcoming the enormous cost of model checking which grows as the product of the number of independent program components, such as, threads of control.-Most researchers agree that abstraction is the key to overcoming this challenge. Research on abstracting the data state of programs using techniques such as predicate abstraction (e.g., [3]) are steadily increasing the size and complexity of programs that can be efficiently analyzed. A complementary approach involves decomposing the program, checking properties of the components, and then composing the analysis results to draw conclusions about the overall behavior of the program. A variety of forms of compositional or modular verification have been studied (e.g., [18]) but they have not been adapted for software written in modern programming languages.

In this paper, we describe automated tool support for adapting existing software model checking frameworks to provide a restricted form of modular verification. Specifically, we consider decomposition of a Java program into two parts: a unit under analysis (henceforth called a unit) and its environment. A unit is any collection of Java classes and its environment consists of the classes with which the unit interacts through the unit's interface ${ }^{1}$. The unit's source code will be the subject of verification along with an abstract model of the environments externally observable behavior. This environment model is derived from specifications written by the user or from the results of analyzing source code that implements environment components. Existing abstraction techniques [9] may be applied to local unit data and to the data that flows between the unit and environment. The resulting abstracted unit and environment may then be analyzed by existing Java model checking frameworks such as JavaPathFinder [30] and Bandera [7].

Thorough treatment of the mechanisms by which the environment may influence the behavior of the unit is essential for sound reasoning. The environment may influence the unit's control (e.g., by invoking methods in the unit's interface or influencing synchronization relationships) and data (e.g., by passing environment data to the unit or by modifying unit data that may flow to the environment). By unit data we mean objects of unit type (i.e., the object's type is included in the unit). Java classes are broken into two categories depending on whether they hold a thread of control. In Java, a class containing the main method or classes that extend(implement) java.lang. Thread (java.lang. Runnable) are labeled active, the rest are termed passive. For consistency, we reuse terminology from previous work [10], and call the active environment classes drivers and passive environment classes stubs. Our approach provides mechanisms by which a wide-range of driver and stub behaviors may be safely ap-

\footnotetext{
'We treat interfaces in Java as classes which comprise a unit interface in our terminology.
} 
proximated.

Experience has shown that the developing environment models for software model checking that are sufficiently precise to enable effective reasoning yet not so overrestrictive that they mask faulty system behaviors is a significant challenge $[19,20]$. Developing such an environment may require an understanding of unstated assumptions about system usage and software interfaces, careful coding to ensure that those assumptions are satisfied in the least restrictive way, and evaluation through model checking of the environment and the unit under analysis. For this reason, we believe that multiple sources of information should be combined to generate environment models that reflect a broad range of realistic execution contexts for a unit under analysis. BEG is aimed at both minimizing the effort required to generate environment models and increasing their fidelity with respect to assumptions about environment behavior. Specifically, BEG currently automates: the discovery of the unit-environment interface based on minimal user supplied information, the synthesis of environment drivers from specifications of the sequences of program actions they may perform, and the synthesis of environment stubs from analysis of the possible program actions executed by existing environment code. Program actions in our setting are statements that may directly influence the data or control state of the unit.

We envision two ways in which environment generation tools can be used effectively: during component development as an adjunct to traditional unit testing approaches and during program validation to enable more efficient reasoning and to model non-source-code components.

During component development individual classes, or groups of classes, that constitute cohesive functional components, perhaps structured as Java packages, may become code complete when the code they interact with (e.g., client code) has not been written. In this setting, the class(es) form a unit and the missing classes they interact with form the environment. To enable effective checking, we expect that developers will need to encode assumptions about the behavior of the environment at the unit's interface. They will need to account for both control and data effects. These assumptions can subsequently be checked against implementations of the missing environment classes as they become code complete.

During program validation when considering a complete application one may break the system into parts to enable more efficient checking of program properties. In this setting, the user selects classes that comprise the unit under analysis and an environment model is automatically extracted. For applications that interact with external entities, such as embedded control software processing data from hardware devices, developers may incorporate assumptions about those interactions to generate a representative model

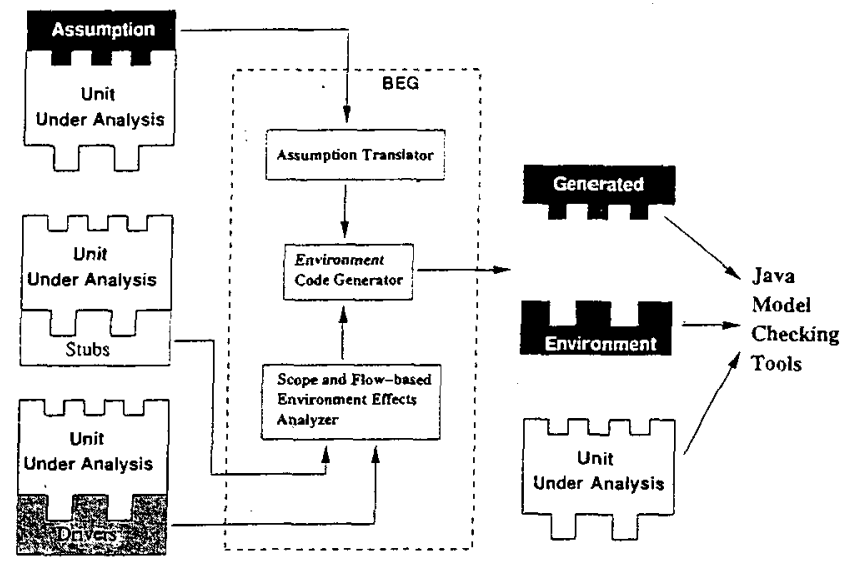

Figure 1. BEG Architecture

of the external environment.

Our approach builds on existing work in assumeguarantee reasoning and in program flow analysis. The approach is implemented in the Bandera Environment Generator (BEG) which supports modular checking of Java source code. Our previous work [28] presented the details of the program analysis and synthesis techniques used to model the data effects of environment implementations. This paper focuses on control effects for active environment components and makes several contributions, including (i) defining a language of program actions with which to specify environment behaviors; (ii) adapting existing specification forms for defining patterns of environment behavior; (iii) synthesing source code models of environment behavior that can be processed by existing model checking frameworks; and (iv) a preliminary evaluation of the effectiveness of BEG in supporting modular source-code model checking. While BEG supports the checking of Java source code, the fundamental concepts it embodies are much more broadly applicable.

The next Section describes our basic approach and an example that is used throughout the paper. Section 3 describes the formalisms for specifying environment behavior and generating environment models as Java source code. Section 4 discusses the soundness of environments relative to specified assumptions. An overview of several case studies using BEG is presented in Section 5. We then compare and contrast our work to existing research in Section 6 and conclude in Section 7.

\section{Basic Approach and An Example}

The fundamental assumption in BEG is that precise reasoning about the unit is desired, but that some precision in modeling the environment may be sacrificed. Our approach is to safely approximate environment data and the environ- 
ment statements that may influence the unit's behavior.

Modelling the effect of environment statements is achieved by a combination of user specifications and analysis of Java source code. Figure 1 shows the architecture of BEG. BEG accepts multiple information sources for generating an environment model. Users identify the unit under analysis by naming the classes, interfaces and packages that comprise the unit. Users provide specifications of their assumptions about the patterns of unit method calls and unit field definitions that the environment may make. If an implementation of the environment is available, BEG may be used to automatically extract the environment assumptions using static analysis techniques. Thus, environment models can be synthesized from a combination of assumption specifications and the results of analyzing implementations. Those models are encoded as Java source code using a collection of modelling primitives to express the atomic execution of environment actions, to encode non-determinism in the environment, and to reflect the approximation in analysis results.

We illustrate our approach - on a small publishsubscribe program implemented using Java's observer and Observable library components. Figure 2 shows class Subject, which is an observable; a field obs of type Buffer, shown on the left side of Figure 4, is a container for watchers that are registered for the Subject. The watcher class contains two bookkeeping fields that record the total number of registration attempts and the number of aborts. Suppose, we are interested in reasoning about whether "Only registered watchers are notified of subject updates". This can be specified in several ways, but one approach is to test whether registered field of Watchers is true at the point where a subject calls update ().

\subsection{Interface Discovery}

The user designates the unit under analysis by naming a collection of Java classes whose properties need to be verified. In general, selection of the classes in the unit is driven by the properties that one wants to reason about.

These classes are analyzed to determine: the fields of unit supertype classes that are referenced in the unit and the non-unit classes that are directly referenced by the unit. Any referenced supertypes are included in the unit. Directly referenced non-unit classes define the unit interface.

For our example and - the mentioned property, subject and watcher should be in the unit. Their supertypes java.util.observable and java.util. Observer and referenced Buffer are in the environment. Note, that the actual environment may consist of more classes due to transitive class and method dependencies, however, BEG identifies classes

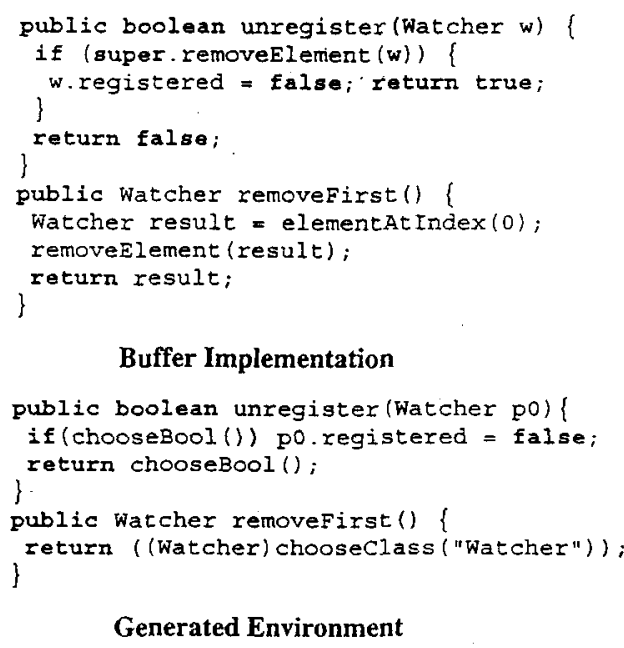

Buffer Implementation

public boolean unregister (Watcher po) ( if (chooseBool ()) po.registered = false; return choosebool () :

Generated Environment

\section{Figure 4. Bounded Buffer Stubs (excerpts)}

that are immediately referenced in the unit. The part of the environment that is invisible to the unit is safely approximated.

\subsection{Driver Specification and Synthesis}

One may specify assumptions about sequences of method calls and unit field definitions that the environment may make on the unit. BEG generates a set of driver threads that implement the most liberal model that is consistent with the given assumptions. Figure 3 illustrates an assumption with one instance of subject and two Watchers and a pair of threads whose behavior is given by regular expressions over method names with parameter values elided; elided parameters means that their value is selected non-deterministically from the possible values of the parameter type. The first thread repeatedly calls the changestate () method on a selected subject and the second calls any sequence of add () or delete() calls on a selected Subject with some watcher.

Figure 3 also shows the generated drivers that capture the assumed behavior. Main allocates the specified instances and starts the execution of the two threads. Thread implementations model the assumption specifications by invoking modeling primitives that capture non-determinism (e.g., chooseBool () chooses among $\{$ true, false $\}$, chooseInt $(n)$ chooses among $\{0, \ldots, n\}$, and chooseclass (C) chooses among the allocated instances of class C) [8].

\subsection{Stub Analysis and Synthesis}

A series of static analyses, including points-to and sideeffects analyses, are applied to determine how the environ- 
The global name scope is defined by annotating instantiations. In an instantiation, the number of instances allocated of a type by the environment is given and those instances may be named. For example, we can adapt the assumption specification in Figure 3 to explicitly name the lone Subject instance, $s$, and reference it regular expression.

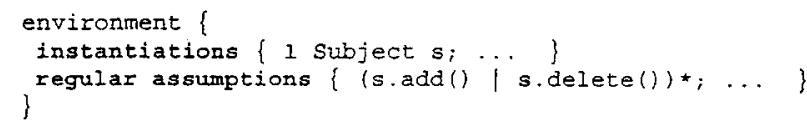

It is important to distinguish between named instances and the set of all instances. The latter is the set of all environment and unit allocated instances. That set forms the universe from which non-deterministic choice primitives over reference types are evaluated.

A local name scope can also be defined that applies to a portion of an assumption specification. The preceding example can be rewritten using a local name scope as:

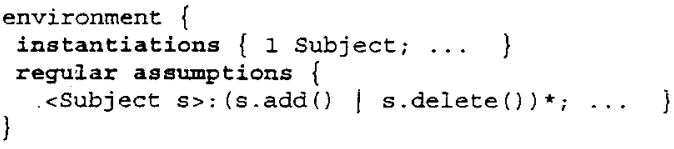

By default local names are bound to a non-deterministically selected value of the given type that holds throughout the name scope (which is denoted explicitly by a pair of \{\} and which extends to the end of the expression by default). Thus, they serve a function similar to universal quantifiers in logics and their primary use is in correlating event occurrences (e.g., that a sequence of actions are applied to the same receiver object).

In these examples, there is a single instance of Subject, thus the three specifications are semantically equivalent. In general, this will not be the case. Local name introduction is interpreted as non-deterministic choice over the the set of allocated instances of the named type, Subject in this case. Local name scopes may be nested and may refer to additional names. For example, $<$ Subject $x>:<$ Subject-x $y>$ :... introduces two names that are guaranteed to refer to distinct instances of Subject. Local names may also be bound to values from the unit. For example, <Ref $x=\operatorname{getRef}()>: x . m()$ introduces a name $x$ that is bound to the value retumed by a call and that is subsequently used to perform a call on method $\mathrm{m}($ ).

\subsection{An Alphabet of Program Actions}

Let $U$ be the set of classes that comprise the unit under analysis and let $B$ denote the set of Java builtin types. We define an alphabet of actions consisting two classes of actions: field assignments and method calls.

Assignments can be either static field assignments or assignments through object references of unit type. Assignments are of the form $r . f=r h s$ where: type $(r) \in U, f$ is of unit type, and rhs is either a scalar constant or Ttype(f), if type $(f) \in B$, or chooseclass (type $(f))$, if type $(f) \in U$. Here $T_{\text {type }} f$ denotes any possible value of type $(f)$; for scalar types the expansion of values is done implicitly via abstraction [9]. The target of the assignment, $r$, is either an introduced name, $T$ for an appropriate type, or the name of a class.

Method call actions are defined using standard Java syntax, but where partial specification of parameters is allowed. Consider a method in class $C$ with signature public $\mathrm{R}$ $m\left(P_{1} p_{1}, P_{2} p_{2}\right)$. We can denote the occurrence of a call to this method with any receiver object of type $C$, a specific value, $v_{1}$, for $p_{1}$, and any value for $p_{2}$ as $m\left(v_{1}, \top\right)$, where $v_{1}$ is an introduced name or a scalar. Partially specified calls may omit the receiver object or any parameter by replacing it with $T$. The meaning of such a call is the set of all calls that can be constructed by replacing $T$ with any legal value of the receiver or parameter type.

We note that $B E G$, through the process of interface discovery, produces the set of program actions (i.e., public method calls and fields at the unit-environment interface) that can be used to define assumptions.

\subsection{Specifying Patterns of Actions}

Regular expressions defined over this alphabet describe a language of actions that can be initiated by the environment. The simplest regular expression is a single program action. Complex environment assumptions are built up using the standard operators for regular expressions: $r$; $s$ (concatenation), $r \mid s$ (disjunction), $r *$ (closure), and $r$ ? (one or more occurrences of $r$ ). Positive closure $(r+)$, bounded iteration $\left(r * n=r_{1} ; r_{2} ; \ldots ; r_{n}\right)$, and a generalization of bounded iteration $(r *\{n, m\}=r * n|r *(n+1)| \ldots \mid r * m)$ are also supported. These expressions can appear in introduced name scopes, where those names are referenced in the program actions used in the expression. The syntax of these assumptions is given in Figure ??. where $a$ is a program action, $a_{f u n}$ are function call actions, $n$ and $m$ are introduced name, and $t$ is a program type name. Legal assumption specifications must also satisfy some type constraints. Specifically, type expressions, te, may only involve types and named variables, $m$, of that type; $m$ here must refer to a name introduced in an enclosing name scope. Similarly, name initializations, $n i$, may only involve function call actions whose type is compatible with the type of the type expression for the introduced name.

As an example, java.util. Iterator presents a simple standard interface for generating the elements in an instance of a container. Semantically, this interface assumes that for each instance of a class implementing the Iterator interface (denoted by the introduced name $i$ ), all clients will call methods in an order that is consistent 


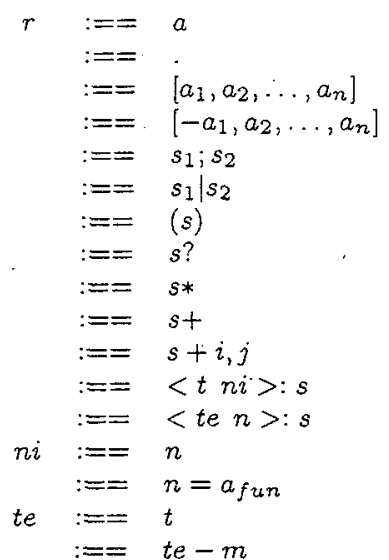

Figure 5. Assumption Syntax

with the following specification:

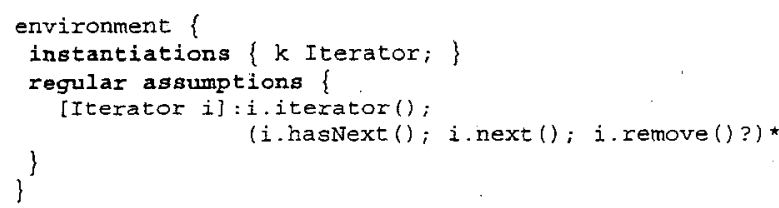

This expresses both required sequencing of calls (e.g., a call to iterator () must precede a call to hasNext) and allowable optional calls (e.g., the occurrence of a single remove () call after a call to next () ) over each instance of Iterator.

\subsection{From Regular Expressions to Code}

Java models of regular expresson assumption specifications can be generated using the templates shown in Figure 6. These templates use the non-deterministic choice constructs mentioned previously and are defined recursively, using code to refer to the code fragment for a given subexpression.

One can view name scope introduction for a subexpression as prefixing a special name binding action to the subexpression. Name scopes are supported by introducing local variables in the body of the driver run () method and assignments that non-deterministically choose an instance to be bound to the name at the point where the name binding action is embedded in the regular expression.

We note that much of the generated model code is internal to the environment. Internal environment states and actions are hidden in our models by embedding them in atomic statements. This has two consequences: internal environment behavior does not contribute to state explosion and internal actions are elided from counter-examples making them shorter and easier to read.

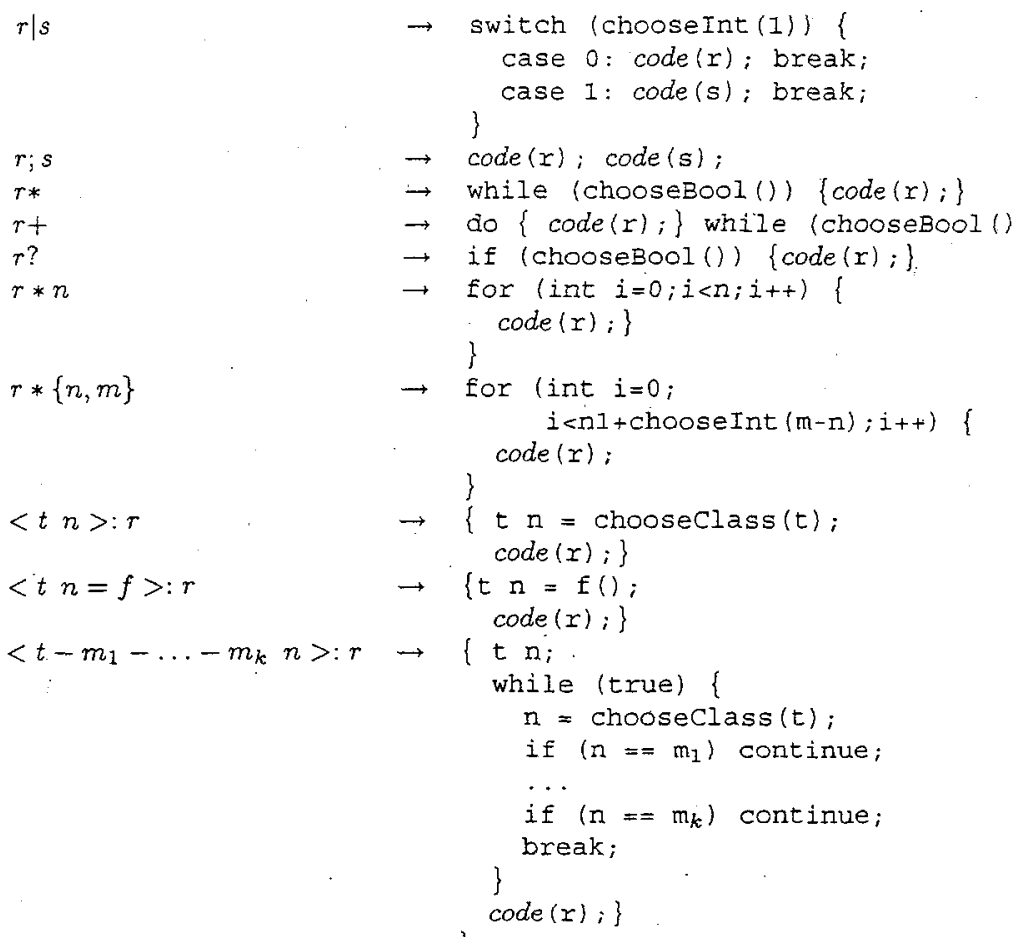

Figure 6. Assumption Semantics

Regular expressions are a familiar formal notation to many developers and our experience is that many find it easier to use than temporal logics. We also support assumptions specified as Linear Temporal Logic (LTL) and generate Java models using an approach that is similar to the one developed for Ada modeling in [22].

\section{Soundness of Synthesized Environments}

In this section, we justify the soundness of synthesized environments with respect to assumption specifications and the results of side-effects analyses.

\subsection{Preliminaries}

Formally, we model the behavior of a concurrent program written in Java as a labelled transition system. Corbett shows how to model the behavior of Java [6] programs as transition systems as defined below, using standard techniques for constructing control flow graphs.

A labelled transition system $P$ is a triple $\langle S(V)$, Act, $R\rangle$, where $V$ is a set of typed program variables, $S(V)$ is the set of states representing valuations of the variables from $V$, Act is an alphabet of actions and $R \subseteq S(V) \times \operatorname{Act} \times S(V)$ is a transition relation. We write 
$s \stackrel{a}{\longmapsto} s^{\prime}$ for $\left(s, a, s^{\prime}\right) \in R$. For a set of variables $W \subseteq V$, $\left.s\right|_{W}$ denotes the valuation of variables from $W$ in state $s$.

States of a system can be regarded as tuples giving the values of all relevant program variables, including the program counter. A transition $s \stackrel{a}{\longmapsto} s^{\prime}$ says that the system can evolve from state $s$ to state $s^{\prime}$ by executing action $a$. The labels on transitions can represent variable assignments, variable tests, and actions modelling transfer of control to and from a procedure; parameter passing can be simulated by communication through common (shared) variables.

We assume that a system is open, i.e. it can interact with its environment through shared variables and actions. If $V$ is the set of variables for an open system, let $V^{\text {int }}$ denote the set of internal (local) variables (that only the system itself may modify) and let $V^{\mathrm{com}}$ denote the set of common (shared) variables, such that $V=V^{\text {int }} \cup V^{\text {com }}$ and $V^{i n t} \cap V^{\text {com }}=\emptyset$. Also if Act is the set of actions of the open system, let Act ${ }^{i n t}$ denote the set of internal actions (a symbol representing an internal action of a system is in the alphabet of only that system) and let Act ${ }^{\text {com }}$ denote the set of communication (or interface) actions, such that $\mathrm{Act}=\mathrm{Act}^{\mathrm{int}} \cup \mathrm{Act} \mathrm{C}^{\mathrm{com}}$ and Act $\mathrm{Ant}^{\mathrm{int}} \cap \mathrm{Act} \mathrm{com}^{\mathrm{com}}=\emptyset$. A system is closed if it may not interact with the environment; a closed system has no shared variables or actions (i.e. $V^{i n t}=\emptyset$ and $\mathrm{Act}^{i n t}=\emptyset$ ).

In order to define the interaction of an open system with the environment, we first define a parallel composition of two systems. Let $P_{1}=\left\langle S\left(V_{1}\right)\right.$, Act $\left._{1}, R_{1}\right\rangle$ and $P_{2}=\left\langle S\left(V_{2}\right), \operatorname{Act}_{2}, R_{2}\right\rangle$ be two open systems. We say that $P_{1}$ and $P_{2}$ are compatible if both their sets of internal variables and sets of internal actions are disjoint (i.e. $V_{1}^{\text {int }} \cap V_{2}^{\text {int }}=\emptyset$ and $\mathrm{Act}_{1}^{\text {int }} \cap \mathrm{Act}_{2}^{\text {int }}=\emptyset$ ).

Let $P_{1}$ and $P_{2}$ be two compatible systems as above. The composition of $P_{1}$ and $P_{2}$, denoted $P_{1} \| P_{2}$, is another system $P=\langle S(V)$, Act,$R\rangle$, where $V=V_{1} \cup V_{2}$, $\mathrm{Act}=\mathrm{Act}_{1} \cup \mathrm{Act}_{2},\left(s, a, s^{\prime}\right) \in R$ iff $\left(\left.a \notin \mathrm{Act}_{i} \wedge s\right|_{V_{i}^{i n t}}=\right.$ $\left.\left.s^{\prime}\right|_{V_{i}^{i n t}}\right) \vee\left(a \in A^{\prime} t_{i} \wedge\left(\left.s\right|_{V_{i}}, a,\left.s^{\prime}\right|_{V_{i}}\right) \in R_{i}\right), i=1,2$.

The two systems synchronize on the shared actions and asynchronously interleave all other actions. The internal variables of system $P_{i}$ may be modified only by the actions of system $P_{i}$, while the common variables may be modified by both systems.

An environment for system $P$ is another system $E$ that is compatible with $P$. Note that after completing the system with a definition of a system representing the environment, the resulting system (i.e. $E \| P$ ) is still open, admitting arbitrary interference from the environment; once we know that all the processes/code modelling the environment have been included, and no further interaction with the extemal world is expected, we may "close" system $E \| P$ by declaring all the shared variables and actions to be internal to the system.

\subsection{Data and Control Effects}

Our program model is general enough to capture different interactions between the system and the environment: through shared data and control (i.e., communication actions); the model does not directly capture dynamic allocation of data, so we put a limit to the number of objects that can flow into the system from the environment.

Our generated environments are either drivers or stubs. Drivers capture the control influences from the environment, while the stubs capture the data influences from the environment.

\subsubsection{Simulation and Preservation Results}

We proceed to define when a system is a sound abstraction of another one. Abstracting means having less details while respecting behaviors of the original system. Let $P=\langle S(V)$, Act,$R\rangle$ and $P^{\prime}=\left\langle S\left(V^{\prime}\right)\right.$, Act $\left.^{\prime}, R^{\prime}\right\rangle$ be two systems. We say that $P$ is a sound abstraction of $P^{\prime}$ iff there is a simulation from $P$ to $P^{\prime}$.

A simulation [18] from $P$ to $P^{\prime}$ is a pair $\left(\rho_{s}, \rho_{a}\right)$ of relations with $\rho_{s} \subseteq S(V) \times S\left(V^{\prime}\right)$ and $\rho_{a} \subseteq$ Act $\times$ Act $^{\prime}$ such that if $\left(s, s^{\prime}\right) \in \rho_{s}$ and $s \stackrel{a}{\longmapsto} t$, then there exists some state $t^{\prime} \in S^{\prime}$ and some action $a^{\prime} \in$ Act' such that $s^{\prime} \stackrel{a^{\prime}}{\longmapsto} t^{\prime}$, $\left(t, t^{\prime}\right) \in \rho_{s}$ and $\left(a, a^{\prime}\right) \in \rho_{a}$. We say that $P$ simulates $P^{\prime}$, denoted $P \preceq P^{\prime}$, if there is a simulation from $P$ to $P^{\prime}$.

When specifying properties of software systems, we use universal temporal logics, i.e., we reason about properties that hold along every possible execution path. A standard result, see e.g. [21], says that simulations preserve satisfaction of formulas of such logics. I.e., if $P \preceq P^{\prime}$, then, for every universal temporal formula $\phi, P^{\prime} \models \phi$ implies $P \models \phi$. However, if $P^{\prime} \models \phi$ does not hold, it does not mean that $P \models \phi$ is necessarily false (i.e., completeness is sacrificed).

Our synthesized environments are sound abstractions of real environments (see $[21,26]$ ), and model checking a system in a synthesized environment is sound. Extending of the results from $[21,26]$, we have the following results: (i) if $E \preceq E_{a b s}$, then $E\left\|P \preceq E_{a b s}\right\| P$, and (ii) if $E \preceq E_{a b s}$ and $P \preceq P_{a b s}$, then $E\left\|P \preceq E_{a b s}\right\| P_{a b s}$. In other words, it is safe to check universal temporal properties in the programs that use the automatically generated environments since these environments are sound abstractions of real environments.

\section{Experience with BEG}

BEG is implemented using the SOOT framework [29]. BEG uses SOOT's symbol table, control flow graph, and bytecode representation, Jimple, to perform its analyses; Jimple is a 3-address SSA-like intermediate form. The tools 
produce Java code as output that includes calls to the modeling methods introduced in Section sec:GENERATE. This section describes our experience applying BEG to generate environments for portions of programs that have appeared recently in the literature on Java verification. The actual verification was performed using either JavaPathFinder or Bandera.

\subsection{Case Studies in Environment Generation}

We have applied BEG to a variety of examples ${ }^{2}$. A number of multi-threaded Java programs that have been the subject of analysis in literature have been re-verified by generating the previously hand-built environments with BEG; the resulting checks are in fact slightly more efficient due to the atomicity of environment behavior in generated environments. In addition to the Observer/Observable example, these examples include: a Producers/Consumers framework for exercising a bounded buffer [15]; RWVSN, Lea's [17] generic readers-writers synchronization framework; and dining philosophers with host, a classic synchronization problem.

While BEG proved to be quite useful in generating environments for these small systems, the tool support is much more valuable when attempting to reason about properties of larger software systems. An increasingly important class of object-oriented software systems are frameworks. Frameworks provide for large-scale reuse of functionality by collecting threads of control, operations and data structures that relate to a specific problem domain (e.g., Swing is a Java framework that supports the development of graphic user interfaces (GUI)). Frameworks present rich interfaces that allow application specific processing to be co-ordinated through the framework. Frameworks are quite difficult to test due to the complexity of their interfaces and the degree of parameterization that is possible to configure their behavior. Current state-of-the-practice in framework testing relies on the use of groups of use cases to drive test case generation. BEG enables the synthesize of drivers that capture multiple framework use cases and mode state machines. Furthermore, the use of non-determinism in assumption specifications allows drivers to span configuration settings. This has the great advantage of allowing configuration-independent properties to be analyzed without having to enumerate combinations of configuration settings.

To explore BEG's support for analyzing frameworks, we consider two non-trivial Java programs. Autopilot is a swing-based GUI for an MD-11 autopilot simulator used for pilot training at NASA [24]; it is a framework client application. ReplicatedWorkers [12], is a parameterizable

\footnotetext{
${ }^{2}$ The details of all examples are given at http://www. cis. ksu. edu/bandera.
}

parallel job scheduling framework. Since neither of these programs can be model checked efficiently in combination with an environment implementation, rather than focus on measures of time and space required for checking, we describe how the tools supported the user in performing modular checking.

\subsection{Autopilot}

The MD-11 autopilot tutor is a web-based application that has a graphical user interface (GUI) that simulates the Autopilot Mode Control Panel and a Primary Flight Display of an MD-11 aircraft autopilot. A user may click on buttons to dial desired altitude and vertical speed, and advance the aircraft towards its goal altitude. Autopilot is implemented as an applet. The application code consists of more than 3600 lines of code clustered in two main classes. These measures bely the true complexity of the system as there is intensive use of java. awt and java. swing GUI frameworks that influences the behavior of the system; in fact the main thread of control is owned by the framework and application methods are invoked as application call-backs.

The system was checked for mode confusion by encoding a model of a pilot's understanding of the aircraft state. That mental model was integrated with the system to monitor GUI inputs. Assertions were inserted to compare the state of system data structures with the state of that model; assertion violations indicated a mismatch between the mental model and the software's state which implies a potential mode confusion.

To analyze the system BEG was used to generate stubs for all the GUI framework components and to generate drivers that encode regular assumptions about pilot behavior. We restrict our attention here to the generation of the drivers, for a more complete description see [27].

The main class of the system is Autopilot which extends java . applet. Applet which in turn extends sev. eral AWT classes. This applet makes a large number of calls to AWT methods in order to create and update the simulated cockpit displays. The properties we wished to reason about, however, were independent of the state of the GUI and we were chose the Autopilot class itself as the unit.

BEG calculated the data effects of the AWT methods called from the Autopilot class and generated safe approximation of the data effects on explicitly defined fields of Autopilot and on fields inherited from AWT classes.

For this system, we found it useful to name the pilot actions to improve the readability of both the assumption specifications and generated counter-examples. As shown in Figure 7, BEG allows one to define mnemonics for GUI interface actions and to define regular assumptions in terms of those mnemonics. Model checking the Autopilot 


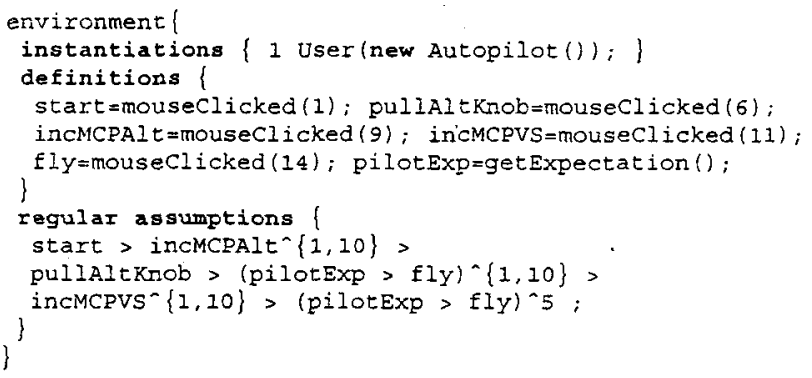

Figure 7. Autopil lot Assumptions

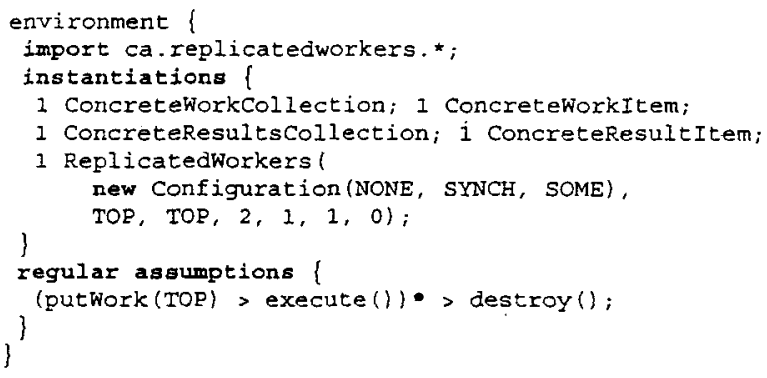

Figure 8. RW Assumptions

class with the generated environment using JavaPathFinder produced the following counter-example:

start $>$ inCMCPAIT^2 $>$ pullAltKnob $>f l \mathrm{Y}^{\wedge} 2>$ inCMCPVS $>f l y$

which indicated a mode confusion anomaly that is possible in the tutor.

It is interesting to note, that a previous effort to build an environment for this application required approximately 6 months and yielded an environment model that was inconsistent with the actual environment implementation. From, relatively simple specifications, BEG generated an environment in less than 4 minutes that is guaranteed to be consistent with the implementation, modulo the fidelity of assumption specifications.

\subsubsection{Replicated Workers}

Replicated Workers (RW) is a configurable framework designed to support the parallelization of simulations. In previous work [10], we applied largely manual techniques to model check a collection of properties of an Ada implementation of this framework. Subsequent to that work the framework was rewritten in Java and has been widely used [12].

Like most frameworks, replicated workers instances create threads internally. Clients control the degree and asynchrony of parallelism in the configuration by passing parameters to the constructor of the framework instance. The replicated workers framework makes significant use of interfaces to enable call-backs to the client supplied computations that are to be parallelized. An environment for the replicated workers, must define and instantiate classes that implement each of the interfaces given in the framework and define appropriate configuration information.

We checked several properties from [10] and were able to reproduce those results with one difference. When checked a framework instance under the environment defined in Figure 8 for deadlock, we found an actual deadlock. The bug was in the Java implementation of a barrier synchronization utility. Its discovery was surprising since the framework has been used in implementing more than ten non-trivial parallel simulation applications and this bug was never discovered. We replaced the barrier implementation with one from java. util. concurrent and the deadlock was eliminated.

\section{Related Work}

Modular approaches to model checking have been studied for more than a decade. This work has been carried out mostly at the theoretical level although there have been some implementations of game-theoretic approaches to reasoning about open systems (e.g., [1]). Our focus is on capturing the complexities of unit/environment interaction that arise in real programming language and supporting the specification and extraction of precise, yet compact environment models.

Environment generation from specifications presented in this paper builds on work of Avrunin et al. [2], who developed tool support for analyzing partially implemented realtime systems with some components implemented in Ada and others described using graphical interval logic and regular expressions, and our previous work on model checking of partial software systems in Ada $[10,11,22]$. In addition to treating Java programs, we support a much richer class of environment specifications and extract environment models from existing code.

Another modular approach to checking multithreaded programs is implemented in Calvin [13]. Their approach is aimed at procedure checking relying on a user specifications of environment assumptions that describe other procedures in the system and constrain interactions among threads. Unlike in our framework, theirs allows for simple invariant specifications and requires that programs obey a restricted class of locking disciplines in interacting.

As complementary to our approach, generation of environment assumptions for optimistic environments has been described in $[14,4]$ Their work is aimed at finding environments within which the unit would satisfy its required properties. This is an important direction to pursue for modular program checking, but we also believe that extraction 
of environments plays an important role when using model checking as a kind of unit testing approach on existing code bases.

There is a number of examples of applying static analysis techniques used in modular analysis or verification. Verisoft incorporates a static analysis to closing of open systems by calculating the influence of extemally defined data [5]. Unlike in our approach, they use a simple notion of data dependence to drive their analysis and do not have the ability to control the precision of the generated system. Stoller [25] describes an approach that computes a partition of a system's inputs based on the data-flow analysis of the system. The idea is to use a single representative input value from each partition to exercise all behaviors of the system and to avoid exercising the same behavior twice. BEG generates environment values based on the user specification or the assumption that the environment data is to be abstracted before model checking phase. Rountev et al. [23] explore how points-to and side-effects analyses may be used to produce summaries for library modules that later may be used for separate analysis of client modules. Unlike in our work, their summries are produced using whole program analysis under the worst-case assumptions about a client and are targeted at the optimizations of the client. Our analyses are modular and explore the information about the unit, if there are call backs from the environment.

\section{Conclusions}

Despite the significant computational complexity of model checking, it has proven effective as an analysis technique that is capable of finding errors in real concurrent Java programs (e.g., the Replicated Workers framework). Modular approaches promise to further scale the application of model checking to software. The Bandera Environment Generator (BEG) provides automated tool support that has proven effective in enabling useful forms of modular analysis.

We are continuing development of the foundations for BEG as well as the tool support. Specifically, we are working on analysis of program lock acquisition to safely approximate the synchronization interaction between the environment and unit. In addition, we are adapting thread modular approaches [13] to enable model checking for arbitrary numbers of environment threads. BEG is being released as part of the Bandera toolset at http://www. cis.ksu. edu/bandera.

\section{Acknowledgments}

The authors would like to thank the members of the Bandera and JPF projects for many helpful discussions and comments related to this work. This work was supported in part by the U.S. Army Research Laboratory and the U.S. Army Research Office under agreement DAAD1901 10564, by DARPAIXO's PCES program through AFRL Contract F33615-00-C3044 , and by Intel Corporation under grant 11462 .

\section{References}

[1] R. Alur, L. de Alfaro, R. Grosu, T. A. Henzinger, M. Kang, C. M. Kirsch, R. Majumdar, F. Mang, and B.-Y. Wang. jmocha: A modelchecking tool that exploits design structure. In Proceedings of the 23rd Annual IEEE/ACM Intemational Conference on Software Engineering, pages pp. 835-836. IEEE Computer Society Press, 2001.

[2] G. S. Avrunin, J. C. Corbett, and L. Dillon. Analyzing partiallyimplemented real-time systems. In Proceedings of the 19 th International Conference on Software Engineering, pages 228-238, 1997.

[3] T. Ball, R. Majumdar, T. Millstein, and S. Rajamani. Automatic predicate abstraction of $C$ programs. In Proceedings of the $A C M$ SIGPLAN 'O1 Conference on Programming Language Design and Implementation (PLDI-O1), volume 36.5 of ACM SIGPLAN Notices, pages 203-213. ACMPress, June 20-22 2001.

[4] J. M. Cobleigh, D. Giannakopoulou, and C. S. Păsăreanu. Learning assumptions for compositional verification. In Tools and Algorithms for the Construction and Analysis of Systems, 9th International Conference (LNCS 2619), Apr. 2003.

[5] C. Colby, P. Godefroid, and L. J. Jagadeesan. Automatically closing open reactive programs. In SIGPLAN Conference on Programming Language Design and Implementation, pages 345-357, 1998.

[6] J. C. Corbett. Constructing compact models of concurrent Java programs. In M. Young, editor, Proceedings of the 1998 International Symposium on Software Testing and Analysis (ISSTA). ACM Press, March 1998.

[7] J. C. Corbett, M. B. Dwyer, J. Hatcliff, S. Laubach, C. S. Păsăreanu, Robby, and $\mathrm{H}$. Zheng. Bandera : Extracting finite-state models from Java source code. In Proceedings of the 22nd International Conference on Software Engineering, June 2000.

[8] J. C. Corbett, M. B. Dwyer, J. Hatcliff, and Robby, Expressing checkable properties of dynamic systems: The bandera specification language. International Joumal on Software Tools for Technology Transfer, 4(1):34-56, 2002.

[9] M. B. Dwyer, J. Hatcliff, R. Joehanes, S. Laubach, C. S. Păsăreanu, Robby, W. Visser, and H. Zheng. Tool-supported program abstraction for finite-state verification. In Proceedings of the 23 rd International Conference on Software Engineering, May 2001.

[10] M. B. Dwyer and C. S. Păsăreanu. Filter-based model checking of partial systems. In Proceedings of the Sixth ACM SIGSOFT Symposium on Foundations of Software Engineering, Nov. 1998.

[11] M. B. Dwyer and C. S. Păsăreanu. Model checking generic container implementations. In Proceedings of the Ist Symposium on Generic Programming, May 1998.

[12] M. B. Dwyer and V. Wallentine. A framework for parallel adaptive grid simulations. Concurrency - Practice and Experience, 9(11):1293-1310, 1997.

[13] C. Flanagan and S. Qadeer. Thread modular model checking. In Model Checking Software (LNCS 2648), May 2003.

[14] D. Giannakopoulou, C. S. Pasareanu, and H. Barringer. Assumption generation for software component verification. In Proceedings of the 17th IEEE Conference on Automated Software Engineering, May 2002.

[15] K. Havelund and T. Pressburger. Model checking Java programs using Java PathFinder. Intemational Journal on Software Tools for Technology Transfer, 1999. 
[16] T. A. Henzinger, R. Jhala, R. Majumdar, and G. Sutre. Lazy abstraction. In Proceedings of the 29 th ACM Symposium on Principles of Programming Languages, pages 58-70, Jan. 2002.

[17] D. Lea. Concurrent Programming in Java[tm], Second Edition: Design principles and Patterns. The Java Series. Addison-Wesley, 2nd edition, 1999.

[18] R. Milner. Communication and Concurrecy. Prentice Hall, 1989.

[19] J. Penix, W. Visser, E. Engstrom, A. Larson, and N. Weininger. Verification of time partitioning in the deos scheduler kernel. In Proceedings of the 22nd International Conference on Software Engineering, June 2000.

[20] C. S. Păsăreanu. Deos kemel: Environment modeling using lt] assumptions. Technical Report Technical Report NASA-ARC-IC2000-196, NASA Ames, 2000.

[21] C. S. Păsăreanu. Abstraction and Modular Reasoning for the Veriffcation of Software. PhD thesis, Kansas State University, 2001.

[22] C. S. Păsăreanu, M. B. Dwyer, and M. Huth. Assume-guarantee model checking of software: A comparative case study. In Theoretical and Applied Aspects of SPIN Model Checking (LNCS 16 80), Sept. 1999.

[23] A. Rountev and B. G. Ryder. Points-to and side-effect analyses for programs built with precompiled libraries. In Proceedings of the 10th International Conference on Compiler Construction (CC'O1), 2001.

[24] L. Sherry, M. Feary, P. Polson, and E. Palmer. Autopilot tutor: Building and maintaining autopilot skills.

[25] S. D. Stoller. Domain partitioning for open reactive systems. In Proceedings of the international symposium on Software testing and analysis, pages 44-54. ACM Press, 2002.

[26] O. Tkachuk. Adapting side effects analysis for modular program model checking. Master's thesis, Kansas State University, 2003.

[27] O. Tkachuk, G. Brat, and W. Visser. Using code level model checking to discover automation surprises. In Proceedings of the 2002 Digital. Avionics Systems Conference, 2002.

[28] O. Tkachuk and M. B. Dwyer. Adapting side effects analysis for modular program model checking. In Proceedings of the Fourth joint meeting of the European Software Engineering Conference and ACM SIGSOFT Symposium on the Foundations of Software Engineering, Sept. 2003.

[29] R. Valle-Rai, L. Hendren, V. Sundaresan, P. Lam, E. Gagnon, and P. Co. Soot - a Java optimization framework. In Proceedings of CASCON'99, Nov. 1999.

[30] W. Visser, K. Havelund, G. Brat, , and S. Park. Model checking programs. In Proceedings of the 15th IEEE Conference on Automated Software Engineering, Sept. 2000. 